\title{
Some Graph Properties of the Optimised Degree Six 3-Modified Chordal Ring Network
}

\author{
R.N. Farah ${ }^{\mathrm{a}}$, S.L.E. Chien ${ }^{\mathrm{a},{ }^{\star}, \text { M. Othman }}{ }^{\mathrm{b}}$ \\ a Department of Mathematics, Faculty of Science and Mathematics, Universiti Pendidikan Sultan Idris, Malaysia \\ ${ }^{b}$ Department of Communication Technology and Networking, Faculty of Computer Science and Technology, Universiti Putra Malaysia, Malaysia \\ * Corresponding author: johnnytan125zr@gmail.com
}

\section{Article history}

Received 9 December 2016

Accepted 22 January 2017

\section{Graphical abstract}

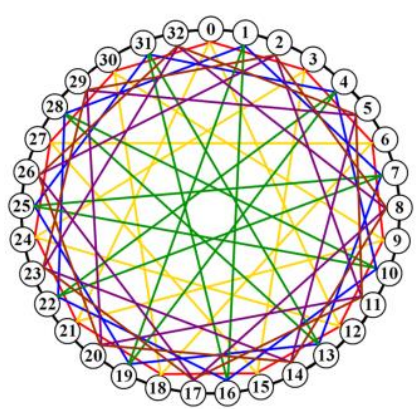

\begin{abstract}
The interconnection topology of a parallel or distributed network is pivotal in ensuring good system performance. It can be modelled by a graph, where its edges represent the links between processor nodes represented by vertices. One such graph model that has gained attention by researchers since its founding is the chordal ring, based on an undirected circulant graph. This paper discusses the degree six 3-modified chordal ring, $\mathrm{CHR6o}_{3}$, and presents its graph theoretical properties of symmetry and Hamiltonicity. $\mathrm{CHR} \mathrm{o}_{3}$ is shown to be asymmetric, and can be decomposed into similar subgraphs, each consisting of only one type of node in its class if ring links are ignored. These properties aid both the development of a routing scheme and determining lower bounds for its chromatic number. Conditions for the existence of a Hamiltonian Circuit within $\mathrm{CHR} \mathrm{o}_{3}$ are also discussed. The existence of a Hamiltonian Circuit within a network simplifies parallel processing as the processors can be arranged to work on a task in a linear array. An Eulerian Circuit was shown to exist in $\mathrm{CHRGo}_{3}$. The existence of an Eulerian Circuit plays a role in routing in optical networks.
\end{abstract}

Keywords: Parallel processing, modified chordal rings, asymmetry, Hamiltonicity, Eulerity.

(C) 2016 Penerbit UTM Press. All rights reserved

\section{INTRODUCTION}

In this age of computing, more and more large networks are designed as parallel or distributive networks. These networks enable the distribution of a task among multiple processor or computing units respectively, such that parts of the task are worked on simultaneously by the processors, thereby reducing system latency. However, the overall performance of the network is greatly attributed to its interconnection topology, which dictates how the processor units are connected to each other, and thus how easily it is for messages to travel between them.

One network topology suited for parallel and distributive networks is the chordal ring, proposed by Arden and Lee in 1981. The chordal ring is modelled after a homogenous undirected circulant graph. Its degree refers to the number of links each node has. Essentially halfway between the ring topology and the complete graph, the chordal ring has the desired properties of both. It is more fault tolerant than a ring due to having multiple paths for which a message from a source node can reach its destination node, and thus, also has lower network diameters than the ring. The application of chordal ring topologies is also more feasible in large networks compared complete graphs. Though, complete graphs have low diameters, the number of links increases exponentially with the number of nodes. This increases the occurrence of bottlenecks when multiple messages simultaneously reach a node, and also incurs a higher cost.

Many researchers have focused on improving and proposing new chordal ring topologies throughout the years since its founding in 1981 due to its favourable parameters of high connectivity, low communication delays, fault tolerance, and symmetry. One such improvement was increasing the degree of the topology, yielding the degree four traditional chordal ring, CHR4 [2]; the degree six traditional chordal ring, CHR6 [3]; and the degree five traditional chordal ring, CHR5 [4]. Altering the connectivity of the chordal ring, resulting in different types of nodes in classes was another way further improving the aforementioned favourable parameters. Examples of these 'modified' chordal rings were the degree six modified chordal ring, CHRm6 [5] along with CHR6ma and CHR6mh [6]; the modified chordal ring of degree three class, $C R c 3$ [7]; the degree five modified chordal ring, CHR5_k [8]; and the degree four modified chordal ring, CHR 4d [9]. Over the years, the properties of the proposed chordal ring topologies such as symmetry [10] as well as Hamiltonicity and asymmetry [11] were also studied over the years, along with compact routing [12], optimum free-table routing [13], and broadcasting [14].

This paper introduces a new degree six chordal ring network topology, the optimised degree six 3-modified chordal ring, $\mathrm{CHR6O}_{3}$ and aims to discuss its properties of symmetry, Eulerity, and Hamiltonicity. The first part of the paper provides an introduction to chordal rings and their contributions as network models, the second part introduces the new degree six modified chordal ring, the third part discusses its graph theoretical properties, and the fourth part concludes the paper.

\section{THE OPTIMISED DEGREE SIX 3-MODIFIED CHORDAL RING, $\mathrm{CHR} \mathrm{OO}_{3}$}

The degree six 3-modified chordal ring is defined as follows:

Definition 1. The optimised degree six 3 -modified chordal ring, $\mathrm{CHR} \mathrm{o}_{3}$, denoted as $\mathrm{CHRGo}_{3}\left(N, s, h_{1}, h_{2}, h_{3}, h_{4}, h_{5}, h_{6}\right)$ is an 
undirected circulant graph. The number of nodes, $N$ as well as all chord lengths $h_{1}, h_{2}, h_{3}, h_{4}, h_{5}$, and $h_{6}$ must be divisible by 3 . Every 3 nodes are grouped into a class.

All the first nodes in the class, $N_{3 i-3}$ (e.g. nodes $\left.0,3,6, \ldots\right)$ are connected together by chords $+h_{1}(\bmod N), \quad-h_{1}(\bmod N)$, $+h_{2}(\bmod N)$, and $-h_{2}(\bmod N)$.

All the second nodes in the class, $N_{3 i-2}($ e.g. nodes $1,4,7, \ldots)$ are connected together by chords $+h_{3}(\bmod N),-h_{3}(\bmod N)$, $+h_{4}(\bmod N)$, and $-h_{4}(\bmod N)$.

All the third nodes in the class, $N_{3 i-1}$ (e.g. nodes $\left.2,5,8, \ldots\right)$ are connected together by chords $+h_{5}(\bmod N), \quad-h_{5}(\bmod N)$, $+h_{6}(\bmod N)$, and $-h_{6}(\bmod N)$, where $i=0,1,2, \ldots . \quad$ Further conditions are that $h_{1}<h_{2}, \quad h_{3}<h_{4}, \quad h_{5}<h_{6} ; \quad$ and $3 \leq$ $h_{1}, h_{2}, h_{3}, h_{4}, h_{5}, h_{6}<\frac{N}{2}$. An example of CHR6o $\mathrm{o}_{3}$ is shown in Fig. 1.

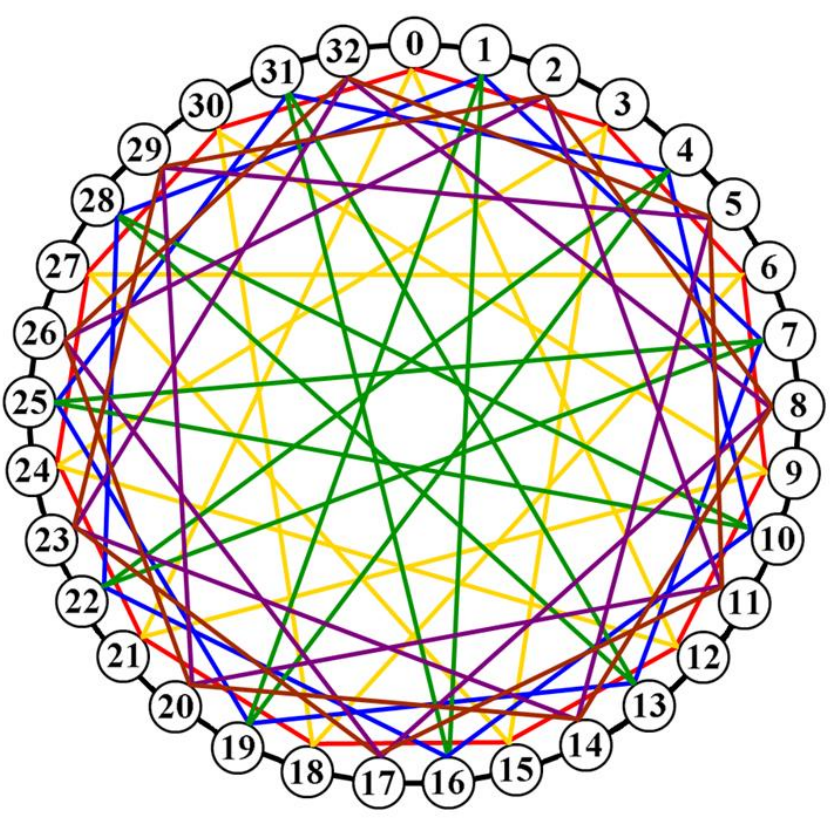

Fig. $1 \mathrm{CHR} \mathrm{o}_{3}(33,1,6,12,3,9,9,12)$

The optimal diameter of $\mathrm{CHR}_{3} \mathrm{o}_{3}$ is given by,

$$
D(G)=\frac{1}{16}\left(17-16 S+8 \sqrt{-4 S^{2}-\frac{109}{64}+\frac{1719}{512 \cdot S}}\right)
$$

where,

$$
S=\sqrt{\frac{1}{192}\left(Q+\frac{4852-576 \cdot N_{d o}}{Q}\right)-\frac{109}{768}}
$$

and,

$$
\begin{aligned}
& Q^{3} \\
& =\left(435103+23544 \cdot N_{d o}\right) \\
& +8 \sqrt{\left(54388+2943 \cdot N_{d o}\right)^{2}-\left(1213-144 \cdot N_{d o}\right)^{3}}
\end{aligned}
$$

The average optimal path length of $\mathrm{CHR} \mathrm{o}_{3}$ for $D(G)>2$ is given by,

$$
\begin{gathered}
d_{\text {avo }}=\frac{1}{5} \cdot \frac{64[D(G)]^{5}-215[D(G)]^{4}+210[D(G)]^{3}}{+260[D(G)]^{2}-229[D(G)]} \\
16[D(G)]^{4}-68[D(G)]^{3} \\
+122[D(G)]^{2}-52[D(G)] \\
\approx \frac{4}{5}[D(G)]+\frac{57}{80}
\end{gathered}
$$

In this model, the processor nodes of a network are represented by the vertices of the graph of $\mathrm{CHR}_{\mathrm{O}} \mathrm{o}_{3}$, and the links connecting them are represented by its edges. In application, each link in the model will be replaced by two links in opposite directions [12].

\section{GRAPH THEORETICAL PROPERTIES OF $\mathrm{CHR6o}_{3}$}

Compared to the degree six traditional chordal ring [3], $\mathrm{CHR} \mathrm{o}_{3}$ is not symmetric. This means that $\mathrm{CHR} \mathrm{o}_{3}$ does not look the same if viewed from different nodes due to different nodes in a class of $\mathrm{CHR} \mathrm{o}_{3}$ having connections of different lengths. According to [11] however, a small symmetry can be seen if nodes with the same types of links are grouped together. This, described as asymmetry, occurs when the chordal ring is neither node-symmetric nor link-symmetric. Nodesymmetry refers to every pair of source and destination nodes being similar.

Theorem 1. $\mathrm{CHR} \mathrm{o}_{3}$ is not node-symmetric.

Proof: Table 1 shows the generalised source and destination nodes for $\mathrm{CHR6o}_{3}$.

Table 1 Source Nodes and their Corresponding Destination Nodes

\begin{tabular}{|c|c|c|c|}
\hline $\begin{array}{c}\text { Source } \\
\text { Nodes }\end{array}$ & \multicolumn{3}{|c|}{ Destination Nodes } \\
\hline$N_{3 i-3}$ & $N_{3 i-3 \pm s}$ & $N_{3 i-3 \pm h_{1}}$ & $N_{3 i-3 \pm h_{2}}$ \\
\hline$N_{3 i-2}$ & $N_{3 i-2 \pm s}$ & $N_{3 i-2 \pm h_{3}}$ & $N_{3 i-2 \pm h_{4}}$ \\
\hline$N_{3 i-1}$ & $N_{3 i-1 \pm s}$ & $N_{3 i-1 \pm h_{5}}$ & $N_{3 i-1 \pm h_{6}}$ \\
\hline & $\begin{array}{c}\text { Destination nodes } \\
\text { with respect to source } \\
\text { nodes which are } \\
\text { similar in each class. }\end{array}$ & $\begin{array}{c}\text { Destination nodes with } \\
\text { respect to source nodes } \\
\text { which are not similar in } \\
\text { each class. }\end{array}$ \\
\hline
\end{tabular}

From the table, it can be seen that the connections of the nodes $N_{3 i-3} \rightarrow N_{3 i-3 \pm s}, N_{3 i-2} \rightarrow N_{3 i-2 \pm s}$, and $N_{3 i-1} \rightarrow N_{3 i-1 \pm s}$ are similar. However, the connections $N_{3 i-3} \rightarrow N_{3 i-3 \pm h_{1}}$ and $N_{3 i-3} \rightarrow N_{3 i-3 \pm h_{2}}$ are not similar with those of the second node in a class of $\mathrm{CHR6O}_{3}$, $N_{3 i-2} \rightarrow N_{3 i-2 \pm h_{3}}$ and $N_{3 i-2} \rightarrow N_{3 i-2 \pm h_{4}}$; and those of the third node in a class of $\mathrm{CHR} \mathrm{o}_{3}, N_{3 i-1} \rightarrow N_{3 i-1 \pm h_{5}}$ and $N_{3 i-1} \rightarrow N_{3 i-1 \pm h_{6}}$. Thus, not every pair of source and destination nodes is similar. Linksymmetry, on the other hand, refers to the similarity of every pair of links between source and destination nodes.

Theorem 2. $\mathrm{CHR6o}_{3}$ is not link-symmetric.

Proof: Table 2 shows the links between source and destination nodes. $s, h_{1}, h_{2}, h_{3}, h_{4}, h_{5}$, and $h_{6}$ are elements of the set of links, $H$.

Table 2 Source Nodes and the Links Leading to their Corresponding Destination Nodes

\begin{tabular}{|c|c|c|c|}
\hline $\begin{array}{c}\text { Source } \\
\text { Nodes }\end{array}$ & \multicolumn{3}{|c|}{$\begin{array}{c}\text { Links to Corresponding Destination Nodes } \\
\text { (Ring Links and Chords) }\end{array}$} \\
\hline$N_{3 i-3}$ & $\pm s$ & $\pm h_{1}$ & $\pm h_{2}$ \\
\hline$N_{3 i-2}$ & $\pm s$ & $\pm h_{3}$ & $\pm h_{4}$ \\
\hline$N_{3 i-1}$ & $\pm s$ & $\pm h_{5}$ & $\pm h_{6}$ \\
\hline
\end{tabular}

For all source nodes, there exist an automorphism $\pi$ of the ring links of $\mathrm{CHRGo}_{3}$ such that $\pi( \pm s)= \pm s$. However, there are no similar automorphisms for the chord lengths: $\pi\left( \pm h_{1}\right)= \pm h_{3}, \pi\left( \pm h_{1}\right)=$ $\pm h_{5}, \pi\left( \pm h_{3}\right)= \pm h_{5}, \pi\left( \pm h_{2}\right)= \pm h_{4}, \pi\left( \pm h_{2}\right)= \pm h_{6}$, and $\pi\left( \pm h_{4}\right)=$ $\pm h_{6}$. Thus, all the chords linking the source nodes of every class to their destination nodes in $\mathrm{CHR6O}_{3}$ are not similar, and $\mathrm{CHRGO}_{3}$ is asymmetric.

Another observation involving symmetry that can be made regarding $\mathrm{CHR} \mathrm{o}_{3}$ is that all the same types of nodes in a class of $\mathrm{CHR} \mathrm{O}_{3}$ are all connected through the same chords. For example, node $N_{3 i-2}$ can only connect to other nodes, $N_{3 j-2}$ through chords $h_{3}$ and $h_{4}$, where $i, j=0,1,2, \ldots$ and $i \neq j$. Connections to other types of nodes in a class of $\mathrm{CHRGo}_{3}$ are achieved through ring links. Without ring links, the three different nodes in a class of $\mathrm{CHRGO}_{3}$ will form disjoint subgraphs consisting of only one type of node in a class. Furthermore, 
since all the chords $h_{1}, h_{2}, h_{3}, h_{4}, h_{5}$, and $h_{6}$ are divisible by 3 , all three different types of nodes in a class of $\mathrm{CHRGo}_{3}$ has essentially the same types of chord connections. Symmetry and asymmetry are one of the advantages of implementing chordal rings as network interconnection toplogy models. Since there are similar nodes, the complexity of routing and broadcasting can be reduced since the similar nodes utilise the same algorithms.

A good measure of connectivity and robustness within a network besides bisection bandwidth is the existence of a Hamiltonian Circuit. A Hamiltonian Circuit in $\mathrm{CHR} \mathrm{oo}_{3}$ visits every node exactly once and returns to its source node. This also implies that links in a particular Hamiltonian Circuit isolated from $\mathrm{CHR6O}_{3}$ are disjoint. Chordal rings may have multiple Hamiltonian Circuits, two by definition in the case of $C H R 4$ [2] and three in the triple loop network of CHR6 [3]. This property makes chordal rings less succeptible to multiple link failures, since the Hamiltonian Circuit may still be maintained [15]. As long as a Hamiltonian Circuit exists in a network, there is still a path from any source node to any destination node for a message to travel, despite increased latency.

Theorem 3. All chord combinations for any $\mathrm{CHR} \mathrm{o}_{3}$ subject to the definition have at least one Hamiltonian circuit.

Proof: All the nodes and ring links of $\mathrm{CHR} \mathrm{o}_{3}$ form a regular graph of degree two whereby all nodes are connected to one another.

Theorem 4. A Hamiltonian circuit of $\mathrm{CHR} \mathrm{o}_{3}$ cannot contain the following in its sequence of links

$$
\begin{array}{llllll}
\text { a. } & \{+s,-s\} & f . & \left\{-h_{2},+h_{2}\right\} & k . & \left\{+h_{5},-h_{5}\right\} \\
\text { b. } & \{-s,+s\} & g . & \left\{+h_{3},-h_{3}\right\} & l . & \left\{-h_{5},+h_{5}\right\} \\
\text { c. } & \left\{+h_{1},-h_{1}\right\} & \text { h. } & \left\{-h_{3},+h_{3}\right\} & \text { m. } & \left\{+h_{6},-h_{6}\right\} \\
\text { d. } & \left\{-h_{1},+h_{1}\right\} & i . & \left\{+h_{4},-h_{4}\right\} & n . & \left\{-h_{6},+h_{6}\right\} \\
\text { e. } & \left\{+h_{2},-h_{2}\right\} & j . & \left\{-h_{4},+h_{4}\right\} & &
\end{array}
$$

Proof: The 'snowflake' geometrical representation in Fig. 2 shows the three different nodes in a class of $\mathrm{CHR} \mathrm{o}_{3}$ represented by three different 'snowflake' models. It can be seen by following the direction of the dendrites, which represent the connectivity of nodes in $\mathrm{CHR6O}_{3}$, that such link combinations cannot exist in a sequence within a Hamiltonian Circuit of $\mathrm{CHR} \mathrm{o}_{3}$. Besides that, such chord combinations are not allowed in a Hamiltonian Circuit because they imply revisiting a node.

Theorem 5. Any Hamiltonian circuit of $\mathrm{CHR} \mathrm{o}_{3}$ must contain some combination of ring links.

Proof: It can be observed that all the same nodes of a class are connected by chords in Fig. 2. For example, a node $N_{3 i-3}$ is connected to nodes, $N_{3 i-3+h_{1}}, N_{3 i-3-h_{1}}, N_{3 i-3+h_{2}}$, and $N_{3 i-3-h_{2}}$. The other 2 nodes in the class, $N_{3 i-2}$ and $N_{3 i-1}$ will change in the same way as their 'snowflake' models, as shown in Fig. 2, are identical but with different chords. Without any ring links, $\mathrm{CHR} \mathrm{O}_{3}$ will be disconnected into 3 node disjoint subgraphs, which cannot exist in a Hamiltonian circuit.

The converse of a Hamiltonian Circuit in $\mathrm{CHRGo}_{3}$ is the Eulerian Circuit. It is a simple path that passes through every link, instead of nodes, in $\mathrm{CHR} \mathrm{o}_{3}$ exactly once and returns to its initial node. Since this path can visit a particular node multiple times, the conditions for its existence in $\mathrm{CHRGo}_{3}$ are less complex than those of the existence of a Hamiltonian Circuit. The existence of an Eulerian Circuit can be determined by node degrees alone. If and only if all nodes in a graph network have even degrees, and if the sum of the degrees of all nodes is an even number, there exists an Eulerian Circuit.

Theorem 6. $\mathrm{CHR} \mathrm{o}_{3}$ contains at least one Eulerian Circuit.

Proof: A graph must only have even degrees and the sum of the degrees of every node must be even to contain at least one Eulerian Circuit, as stated by Leonhard Euler in the Seven Bridges of Königsberg problem. $\mathrm{CHRGO}_{3}$ is a homogenous interconnection topology where all nodes have the same connectivity i.e. the same degree of 6 , which implies it is modelled after a 6-regular graph. There are no nodes with odd degrees, and the sum of all the degrees of all vertices is $6 N .6 N$ is always an even number regardless of whether the network size is even or odd.

The concept of Eulerity plays several key roles in parallel processing as it maps out disjoint paths within a network. The ordering of logic gates in a network is optimised through Eulerity [16]. Furthermore, in the case where the same message is routed through an optical network through all-to-all routing, the message will be assigned the same wavelength and needs to travel through link-disjoint paths [17].
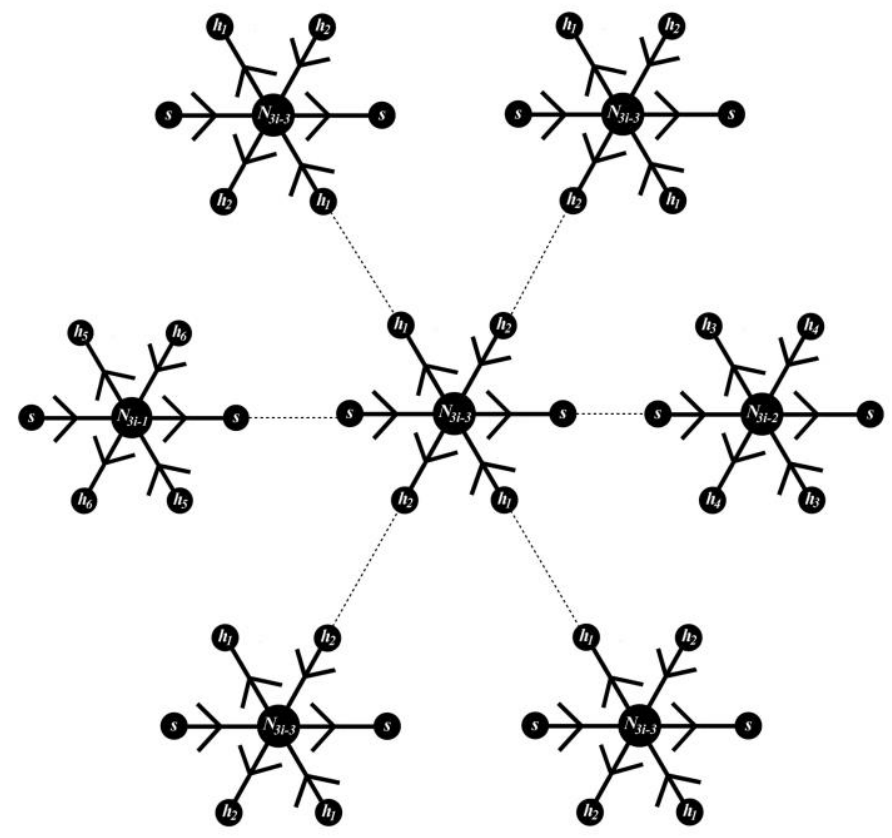

Fig. 2 The 'Snowflake' Geometrical Representation of $\mathrm{CHRGO}_{3}$

\section{CONCLUSIONS}

A new degree six modified chordal ring was introduced in this paper and some of its graph theoretical properties were discussed. The degree six 3-modified chordal ring, $\mathrm{CHR} \mathrm{o}_{3}$ was shown to be asymmetric and conditions for the existence of a Hamiltonian Circuit within its networks were presented and justified. These properties are important in developing a routing scheme for $\mathrm{CHR} \mathrm{o}_{3}$. An Eulerian Circuit was also shown to exist in $\mathrm{CHR6o}_{3}$. Eulerity is important for network applications which require link-disjoint paths, such as optical routing of the same message.

\section{REFERENCES}

[1] B.W. Arden, H. Lee. Analysis of Chordal Ring Network. IEEE Trans. Computer, C-30(4) (1981) 291-295.

[2] R.F. Browne, R.M. Hodgson. Symmetric Degree-four Chordal Ring Networks. IEE, Computers and Digital Techniques, (1990) 310-318.

[3] A.A. Matroud. Communication in Chordal Ring Networks. Ph.D Thesis. Universiti Putra Malaysia. Malaysia. (2006).

[4] S. Bujnowski, B. Dubalski, A. Zabludowski, J.M. Pedersen, T. Riaz. Analysis of Degree 5 Chordal Rings for Network Topologies. Image Processing \& Communications Challenges 3, Advances in Intelligent Systems and Computing, 102 (2011) 445-457.

[5] R.N.F Azura, M. Othman, M.H. Selamat, Y.H. Peng. Modified Degree Six Chordal Rings Network Topology. Simposium Kebangsaan Sains Matematik ke-16. (2008) 515-522.

[6] S. Bujnowski, B. Dubalski, A. Zabludowski, D. Ledziński, T. Marciniak, J.M. Pedersen. Comparison of Modified Degree 6 Chordal Rings. Image Processing \& Communications Challenges 2, Advances in Intelligent Systems and Computing, 84 (2010) 435-445. 
[7] N. Irwan, R.N. Farah, A.Z.M. Sofi. Network Properties for Classes of Chordal Ring Degree Three Topology. International Conference on Intelligent Network and Computing. (2010) 16-20.

[8] B. Dubalski, S. Bujnowski, D. Ledziński, A. Zabłudowski,P. Kiedrowski. Analysis of Modified Fifth Degree Chordal Rings. New Frontiers in Graph Theory. China: InTech. (2012) 43-88.

[9] D. Ledziński, S. Bujnowski, T. Marciniak, J.M. Pedersen, J.G. Lopez. Network Structures Constructed on Basis of Chordal Rings 4th Degree. Image Processing and Communications Challenges 5, Advances in Intelligent Systems and Computing, 233 (2014) 281-299.

[10] L. Barrière. Symmetry Properties of Chordal Rings of Degree 3. Discrete Applied Mathematics, 129 (2003) 211-232.

[11] R.N.F. Azura, M. Othman, Y.H. Peng, M.H. Selamat. On Properties of Modified Degree Six Chordal Rings Network. Malaysian Journal of Mathematical Sciences, 4(2) (2010) 147-157.

[12] L. Narayanan, J. Opatrny. Compact routing on chordal rings of degree four. Algorithmica, 23 (1999) 72-96.

[13] R.N. Farah, M. Othman, M.H. Selamat. An Optimum Free-Table Routing Algorithms of Modified and Traditional Chordal Ring Networks of Degree Four. Journal of Materials Science and Engineering, 4(10) (2012) 78-89.

[14] R.N. Farah, M. Othman. Broadcasting Communication in High Degree Modified Chordal Ring Networks. Applied Mathematics \& Information Sciences, 8(1) (2014) 229-233.

[15] B. Parhami. Chordal Rings Based on Symmetric Odd-Radix Number Systems. The 2005 International Conference on Communications in Computing, (2005) 196-199.

[16] R. Kuntal. Optimum Gate Ordering of CMOS Logic Gates Using Euler Path Approach: Some Insights and Explainations. Journal of Computing and Information Technology 15(1) (2007) 85-92.

[17] L. Narayanan, J. Opatrny, D. Sotteau. All-To-All Optical Routing in Chordal Rings of Degree 4. Algorithmica, 31 (2001) 155-178. 